\title{
Momento e Frequência das Visitas de Pré-Natal: Repercussões Sobre os Nascimentos Pré-Termo
}

\author{
Timing and Frequency of Prenatal Visits: \\ Impact on the Preterm Delivery \\ Edson Nunes de Morais, Taciana L. Alflen, \\ Patricia Spara, Patrícia El Beitune
}

\begin{abstract}
RESUMO
O objetivo deste estudo foi determinar a eficácia da assistência pré-natal, com respeito às visitas (número e momento da primeira), sobre a idade gestacional e peso fetal ao nascimento. Avaliamos prospectivamente o efeito da assistência pré-natal sobre um grupo de 648 recémnascidos no Hospital Universitário de Santa Maria de forma consecutiva, de gestações com idade entre 28 e 40 semanas. Os nascimentos de fetos pré-termo (< 37 semanas) corresponderam a $17,7 \%$ de todos os nascimentos; os recém-nascidos de baixo peso $(<2.500 \mathrm{~g})$ a 20,5\% e os recém-nascidos de muito baixo peso $(<1.000 \mathrm{~g})$ a 2,8\%. Quando a primeira visita pré-natal foi realizada antes da $12^{a}$ semana de gestação, somente $5,1 \%$ dos neonatos nasceram antes da $37^{a}$ semana de idade gestacional ou tiveram peso ao nascimento $<2.500 \mathrm{~g}$. Entretanto, quando a primeira visita foi realizada após a $28^{a}$ semana, a porcentagem de nascimentos pré-termo foi de 41,3\% e o peso fetal inferior a $2.500 \mathrm{~g}$, de 43,5\%. Observou-se uma associação significante entre o aumento do número de visitas prénatais, cuidados precoces e o decréscimo dos nascimentos pré-termo e de fetos de baixo peso. Concluímos que a maior freqüência de visitas pré-natais e a assistência precoce à gestante podem reduzir as taxas de nascimentos pré-termo e de fetos de baixo peso.
\end{abstract}

PALAVRAS-CHAVE: Pré-natal. Prematuridade. Recém-nascidos de baixo peso. Peso fetal.

\section{Introdução}

É aceito universalmente que os cuidados antenatais têm um efeito benéfico sobre os resultados perinatais. Entretanto, não está claro se a freqüência das visitas pré-natais e o início precoce das mesmas têm repercussões sobre as complicações da gestação e o peso fetal ao nascimento.

A importância da assistência pré-natal sobre a saúde da gestante e seu concepto é aceita pela

Departamento de Ginecologia e Obstetrícia do Centro de Ciências da Saúde da Universidade Federal de Santa Maria Correspondência:

Edson Nunes de Morais

Av. Borges de Medeiros, 1699/604

97015-090 - Santa Maria - RS. grande maioria dos autores. Estudos têm demonstrado que uma política de assistência pré-natal bem executada, leva à minimização de taxas de mortalidade e morbidade perinatal ${ }^{9}$, bem como dos índices de partos pré-termo ${ }^{10}$. No entanto, nem todos assim pensam. Para Chadwick ${ }^{3}$ a mortalidade perinatal é alta nas classes sociais baixas e a assitência antenatal não tem se modificado ao longo deste século, permanecendo de forma monótona, sob uma mesma rotina. Refere ainda, que ao tema é dado pouca importância e as pesquisas e avaliações são realizadas infreqüentemente.

Quando se discute o significado da assistência pré-natal para a gestante e seu concepto, um dos pontos que merece a atenção dos pesquisadores diz respeito ao momento ideal para a realização da primeira consulta e o número 
desejável de visitas pré-natais ${ }^{19}$.

A freqüência das visitas antenatais bem como o momento da primeira visita, são usualmente referidos como prováveis elementos contribuintes para as taxas de mobimortalidade perinatal. A OMS preconiza que a gestante deve realizar pelo menos 5 consultas durante a gravidez. Alguns autores entendem que menos que 10 visitas pré-natais pode fazer diferença nos resultados perinatais, em particular no que se refere ao nascimento de fetos pré-termo ${ }^{8}$. Uma assistência pré-natal adequada significa precocidade no tratamento, assiduidade nas visitas e boa qualidade de atendimento.

Com o objetivo de avaliar se o número de visitas pré-natais e o momento da primeira visita repercutem sobre o nascimento de fetos pré-termo e o peso fetal ao nascimento, o presente trabalho foi levado a efeito.

\section{Material e métodos}

O material do presente trabalho consiste de dados obtidos prospectivamente de 648 pacientes, portadoras de gestações simples, atendidas no Serviço de Obstetrícia do Hospital Universitário de Santa Maria, no período de agosto a dezembro de 1995. As pacientes estudadas apresentavam gestações de baixo, médio e alto risco.

Analisamos a idade gestacional em semanas, e o peso fetal em gramas, associando-os ao número de consultas pré-natais e momento da primeira visita. As pacientes foram divididas, inicialmente, quanto à idade gestacional em 5 grupos de estudo: $<28$ semanas, 28 a 31,32 a 36,37 a 40 e mais de 40 semanas. Quanto ao peso fetal, as gestantes foram divididas também em 5 grupos: peso fetal de $<1.000 \mathrm{~g}$, entre 1.000 e $1.499 \mathrm{~g}, 1.500 \mathrm{e}$ $2.499 \mathrm{~g}, 2.500$ e $3.999 \mathrm{~g} \mathrm{e}^{3} 4.000 \mathrm{~g}$. A idade gestacional foi calculada levando-se em conta a data da última menstruação e exames de ultrasonografia.

Quanto à realização de pré-natal dividiu-se as pacientes nos seguintes grupos: nenhuma consulta, de 1 a 2 consultas, de 3 a 5 , de 6 a 9 e acima de 9 consultas. Quanto à época da primeira visita pré-natal (semanas de gestação), os grupos foram divididos em: antes da $12^{\mathrm{a}}$ semana, entre a $12^{\mathrm{a}}$ e $16^{\mathrm{a}}, 17^{\mathrm{a}}$ e $20^{\mathrm{a}}, 21^{\mathrm{a}}$ e $28^{\mathrm{a}}$ e após a $28^{\mathrm{a}}$ semana.

Posteriormente dividiu-se as gestantes quanto à idade gestacional e peso fetal ao nascimento, em função do número de consultas e momento da primeira visita pré-natal, em dois grupos: com idade gestacional $<37$ semanas e $\geq 37$ semanas, e com peso fetal $<2.500 \mathrm{~g}$ e $\geq 2.500 \mathrm{~g}$, respectivamente.

Das 648 gestantes estudadas, em 128 casos não se obteve informações quanto ao momento da realização da primeira consulta pré-natal. Foram consideradas para análise apenas os dados completos de 520 pacientes.

As gestantes que realizaram pré-natal o fizeram no Serviço de Obstetrícia do HUSM ou em postos da rede do Sistema Único de Saúde do município de Santa Maria, cujas rotinas são semelhantes.

Para o estudo da possivel associação entre as variáveis estudadas, empregou-se o teste não paramétrico do qui quadrado observando-se um nível de significância de $1 \%$.

\section{Resultados}

Os resultados do presente trabalho serão apresentados sob forma de Tabelas (de 1 a 4) e Figuras (de 1 a 4). A Tabela 1 mostra a distribuição de 648 nascimentos em função do número de consultas pré-natal e as diversas faixas de idade gestacional estudadas. Observa-se que à medida que aumenta o número de visitas pré-natal, maior é a idade gestacional dos fetos ao nascimento. Quando a paciente não realizou nenhuma visita, apenas $63,6 \%$ delas tiveram seus filhos com idade gestacional superior a 37 semanas, ao passo que o número de visitas sendo igual ou superior a 6 , os fetos a termo totalizaram mais de $90 \%$.

Na Tabela 2 encontra-se a distribuição de 648 nascimentos em função do número de consultas pré-natais e as diversas faixas de peso fetal ao nascimento. Estes dados demonstram que também sobre o peso fetal repercutiu o número de visitas pré-natais. Quando as gestantes não fizeram nenhuma visita pré-natal, ou menos de 3 , a porcentagem de fetos de baixo peso foi de $45,0 \%$, ao passo que se a gestante realizava 6 ou mais consultas esse porcentual diminui para $8,07 \%$. Na Tabela 3 estão relacionados 519 nascimentos segundo o momento da primeira consulta pré-natais e as diversas faixas de idade gestacional estudadas.

O momento da primeira consulta pré-natal refletiu-se na freqüência de nascimentos pré-termo, isto é, quanto mais precoce a primeira consulta menor o número de nascimento prematuros. Em apenas 12 casos $(5,06 \%)$ de 237 , em que a primeira visita se deu antes da $12^{\mathrm{a}}$ semana, os fetos nasceram antes da $37^{\mathrm{a}}$ semana de gestação. Quando a primeira visita ocorreu após a $20^{\mathrm{a}}$ semana, em $44(37,0 \%)$ de 119 casos, ocorreu o nascimento de fetos pré-termo. Na Tabela 4 encontram-se os dados de 519 nascimentos em função do momento da primeira consulta pré-natal e as diversas faixas de peso fetal estudadas. Estes 
dados demonstram que quanto mais precoce o momento da primeira visita pré-natal, tanto menor o número de nascimentos de fetos de baixo peso. A primeira visita pré-natal ocorrendo antes da $12^{\mathrm{a}}$ semana de gestação, apenas 5,1\% dos fetos nasceram com peso inferior a $2.500 \mathrm{~g}$, ao passo que $40,3 \%$ tiveram este peso se a primeira consulta ocorreu após a $20^{\mathrm{a}}$ semana.

Foi encontrada uma associação significante ( $p<0,01)$ entre a idade gestacional de 648 recémnascidos (<37 semanas e >37 semanas), e o número de consultas pré-natal (Figura 1). O peso de 648 recém-nascidos ( $<2.500 \mathrm{~g} \mathrm{e}>2.500 \mathrm{~g})$ associou-se significantemente $(\mathrm{p}<0,01)$ com o número de consultas pré-natais, como demonstra a Figura 2. A Figura 3 mostra a associação significante $(p<0,01)$ entre o momento da primeira visita pré-natal e a idade gestacional em semanas $(<37$ semanas e $>37$ semanas) de 519 nascimentos, enquanto a Figura 4 ilustra a distribuição de 519 recém-nascidos em função do momento da primeira consulta pré-natal e o peso fetal ao nascimento $(<2.500 \mathrm{~g}$ e $>2.500 \mathrm{~g})$, observamos uma associação significante entre estas variáveis $(\mathrm{p}<0,01)$.

Tabela 1 - Distribuição de 648 nascimentos em função do número de consultas pré-natais (PN) e a idade gestacional (IG) ao nascimento

\begin{tabular}{|c|c|c|c|c|c|c|c|c|c|c|c|c|}
\hline $\begin{array}{l}\text { IG (semanas) } \\
\text { Consultas PN }\end{array}$ & \multicolumn{2}{|c|}{$\begin{aligned}<28 \\
\text { № }-\%\end{aligned}$} & \multicolumn{2}{|c|}{$\begin{array}{c}28-31 \\
\text { № }-\%\end{array}$} & \multicolumn{2}{|c|}{$\begin{array}{c}32-36 \\
\text { № }-\%\end{array}$} & \multicolumn{2}{|c|}{$\begin{array}{c}37-40 \\
\text { № }-\%\end{array}$} & \multicolumn{2}{|c|}{$\begin{array}{c}>40 \\
\text { № }-\%\end{array}$} & \multicolumn{2}{|c|}{$\begin{array}{l}\text { TOTAL } \\
\text { № - \% }\end{array}$} \\
\hline Nenhuma & 8 & 6,2 & 10 & 7,8 & 29 & 22,5 & 58 & 45,0 & 24 & 18,6 & 129 & 19,0 \\
\hline $1-2$ & 2 & 2,4 & 13 & 15,9 & 7 & 8,5 & 46 & 56,1 & 14 & 17,1 & 82 & 12,6 \\
\hline $3-5$ & 4 & 2,3 & 2 & 1,1 & 18 & 10,2 & 96 & 54,2 & 57 & 32,2 & 177 & 27,3 \\
\hline $6-9$ & 2 & 1,0 & 1 & 0,5 & 15 & 7,2 & 102 & 49,0 & 88 & 42,3 & 208 & 32,1 \\
\hline$\geq 10$ & 0 & 0,0 & 0 & 0,0 & 6 & 1,0 & 40 & 76,9 & 6 & 11,0 & 52 & 8,0 \\
\hline TOTAL & 16 & 2,5 & 26 & 4,0 & 75 & 11,6 & 342 & 52,8 & 189 & 29,2 & 648 & 100,0 \\
\hline
\end{tabular}

Tabela 2 - Distribuição de 648 nascimentos em função do número de consultas pré-natais (PN) e o peso fetal (g) ao nascimento

\begin{tabular}{|c|c|c|c|c|c|c|c|c|c|c|c|c|}
\hline Peso fetal (g) & \multicolumn{2}{|c|}{$\begin{array}{l}<1.000 \\
№-\infty\end{array}$} & \multicolumn{2}{|c|}{$1.000-1.499$} & \multicolumn{2}{|c|}{$1.500-2.499$} & \multicolumn{2}{|c|}{$2.500-3.999$} & \multicolumn{2}{|c|}{$\geq 4.000$} & \multicolumn{2}{|c|}{ TOTAL } \\
\hline Nehuma & 10 & 7,8 & 8 & 6,2 & 35 & 27,1 & 67 & 51,9 & 9 & 7,0 & 129 & 19,0 \\
\hline $1-2$ & 6 & 7,3 & 5 & 6,1 & 31 & 37,8 & 28 & 34,1 & 12 & 14,6 & 82 & 12,6 \\
\hline $3-5$ & 2 & 1,1 & 3 & 1,7 & 12 & 6,8 & 151 & 85,3 & 9 & 5,1 & 177 & 27,3 \\
\hline TOTAL & 18 & 2,8 & 17 & 2,6 & 98 & 15,1 & 471 & 72,7 & 44 & 6,8 & 648 & 100,0 \\
\hline
\end{tabular}

Tabela 3 - Distribuição de 519 nascimentos em função do momento da $1^{\mathrm{a}}$ consulta pré-natal e a idade gestacional (IG) ao nascimento

\begin{tabular}{|c|c|c|c|c|c|c|c|c|c|c|c|c|}
\hline \multirow{2}{*}{$\begin{array}{l}\text { IG (semanas) } \\
\text { Primeira } \\
\text { consulta (sem) }\end{array}$} & \multicolumn{2}{|c|}{$<28$} & \multicolumn{2}{|c|}{$28-31$} & \multicolumn{2}{|c|}{$32-36$} & \multicolumn{2}{|c|}{$37-40$} & \multicolumn{2}{|c|}{$>40$} & \multicolumn{2}{|c|}{ TOTAL } \\
\hline & $\mathrm{N}$ & $-\%$ & $\mathrm{~N}$ & $-\%$ & № & $-\%$ & № & $\%$ & № & $-\%$ & & $-\%$ \\
\hline$<12$ & 0 & 0,0 & 1 & 0,42 & 11 & 4,6 & 145 & 61,2 & 80 & 33,8 & 237 & 45,6 \\
\hline $12-16$ & 0 & 0,0 & 1 & 1,0 & 4 & 4,0 & 53 & 53,5 & 41 & 41,5 & 99 & 19,1 \\
\hline $17-20$ & 0 & 0,0 & 1 & 1,5 & 8 & 12,3 & 37 & 56,9 & 19 & 29,2 & 65 & 12,5 \\
\hline TOTAL & 8 & 1,5 & 16 & 3,1 & 46 & 8,8 & 285 & 54,8 & 165 & 31,8 & 520 & 100,0 \\
\hline
\end{tabular}


Tabela 4 - Distribuição de 519 nascimentos em função do momento da $1^{\mathrm{a}}$ consulta pré-natal e o peso fetal (g) ao nascimento.

\begin{tabular}{|c|c|c|c|c|c|c|c|c|c|c|c|c|}
\hline $\begin{array}{l}\text { Peso fetal (g) } \\
\text { Primeira } \\
\text { consulta (sem). }\end{array}$ & \multicolumn{2}{|c|}{$\begin{array}{l}<1.000 \\
\text { № }-\%\end{array}$} & \multicolumn{2}{|c|}{$\begin{array}{c}1.000-1.499 \\
\text { № }-\%\end{array}$} & \multicolumn{2}{|c|}{$\begin{array}{c}1.500-2.499 \\
\text { № }-\%\end{array}$} & \multicolumn{2}{|c|}{$\begin{array}{c}2.500-3.999 \\
\text { № - \% }\end{array}$} & & $\begin{array}{l}.000 \\
-\quad \%\end{array}$ & $\begin{array}{l}\mathrm{TC} \\
\text { № }\end{array}$ & $\begin{array}{l}\text { TAL } \\
-\%\end{array}$ \\
\hline$<12$ & 0 & 0,0 & 1 & 0,4 & 11 & 4,6 & 216 & 91,1 & 9 & 3,9 & 237 & 45,6 \\
\hline $12-16$ & 0 & 0,0 & 1 & 1,0 & 12 & 12,1 & 81 & 81,8 & 5 & 5,1 & 99 & 19,1 \\
\hline $17-20$ & 1 & 1,5 & 2 & 3,0 & 4 & 6,0 & 46 & 70,1 & 12 & 18,5 & 65 & 12,5 \\
\hline $21-28$ & 3 & 4,1 & 2 & 2,7 & 23 & 31,5 & 42 & 57,5 & 3 & 4,2 & 73 & 14,0 \\
\hline$>28$ & 4 & 8,7 & 3 & 6,5 & 13 & 28,3 & 20 & 43,5 & 6 & 13,0 & 46 & 8,8 \\
\hline TOTAL & 8 & 1,5 & 9 & 1,7 & 63 & 12,2 & 405 & 77,9 & 35 & 6,7 & 520 & 100,0 \\
\hline
\end{tabular}

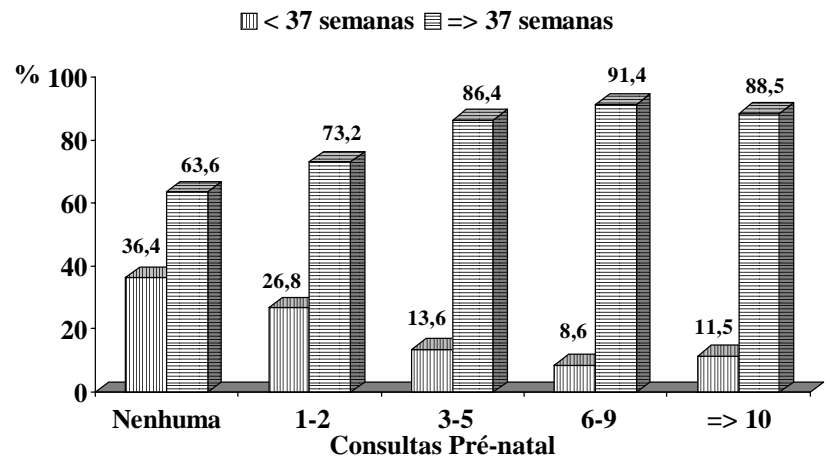

Figura 1 - Distribuição de 648 nascimentos em função do número de consultas pré-natal e a idade gestacional $<37 \mathrm{e} \geq 37$ semanas ao nascimento.

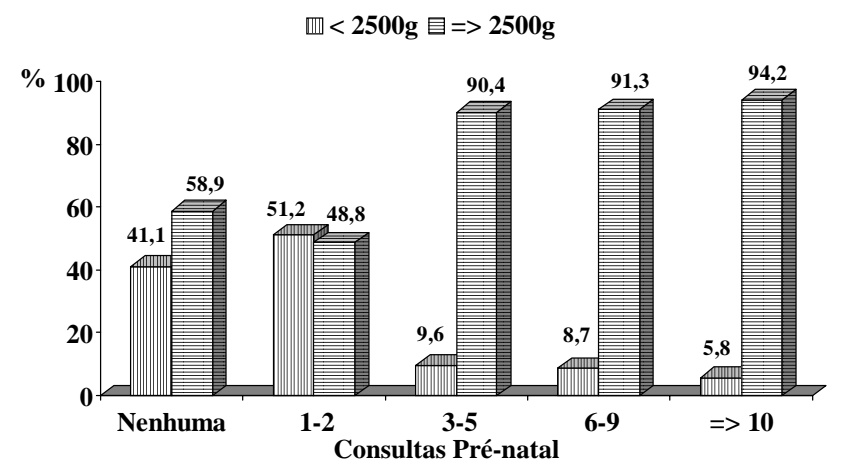

Figura 2 - Distribuição de 648 nascimentos em função do número de consultas pré-natal e o peso fetal $<2.500 \mathrm{~g} \mathrm{e} \geq 2.500 \mathrm{~g}$ ao nascimento.

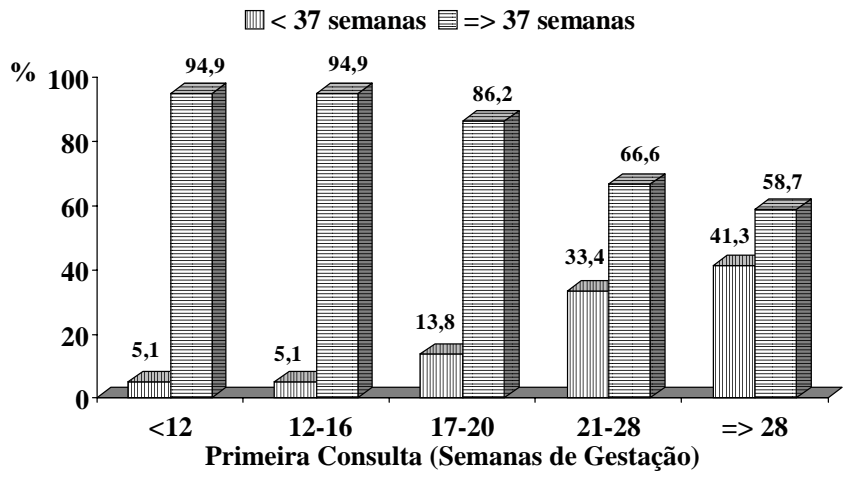

Figura 3 - Distribuição de 519 nascimentos em função do momento da $1^{\mathrm{a}}$ consulta pré-natal e a idade gestacional $<37$ e $\geq 37$ semanas ao nascimento.

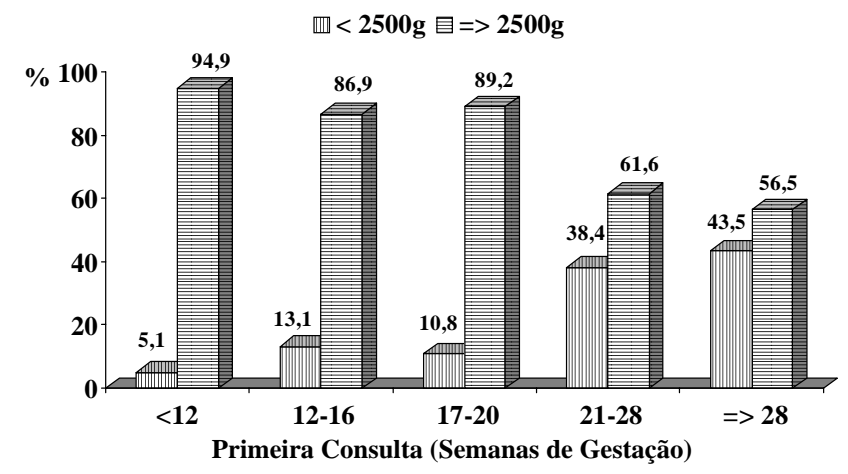

Figura 4 - Distribuição de 519 nascimentos em função do momento da $1^{\mathrm{a}}$ consulta pré-natal e o peso fetal $<2.500 \mathrm{~g}$ e $\geq 2.500 \mathrm{~g}$ ao nascimento. 


\section{Discussão}

Não há dúvida que uma boa assistência prénatal tem efeitos positivos sobre as taxas de morbidade e mortalidade perinatais e tal afirmativa tem sido demonstrada por inúmeros autores. ${ }^{7,12,13,17}$ A mortalidade perinatal na Alemanha caiu nas últimas 4 décadas de $5 \%$ para $0,5 \%$ em 1992. Esta queda se deu em função da melhoria das organizações ligadas à saúde, tais como movimento para os partos serem realizados em ambiente hospitalar, leis de proteção à gestante, guia de princípios da assistência pré-natal, regionalização dos casos de alto risco, e incremento na monitorização fetal ante e intraparto. Dentre estes últimos arrolam-se a ultra-sonografia e cuidados intensivos neonatais, ventilação assistida e uso de surfactante pulmonar ${ }^{9}$.

Um dos principais problemas da perinatologia atual continua sendo o nascimento pré-termo, cujas taxas de morbidade e neomortalidade ainda preocupam os obstetras e neonatólogos.

Vários são os fatores responsabilizados pelo nascimento de fetos pré-termo. Entre eles citamse a idade materna, acima de 35 anos e abaixo de 20, educação inferior a 10 anos, gestantes solteiras, presença de patologias maternas, consumo acima de 10 cigarros/dia, e assistência pré-natal inadequada. Outros fatores como a etinicidade, parecem ter influência nos nascimentos pré-termo. Nos Estados Unidos os nascimentos pré-termo de mulheres porto-riquenhas prevalecem sobre os das cubanas, mexicanas, centro e sul-americanas, dominicanas e de outras etnias ${ }^{4}$.

Não há consenso entre os autores, se algumas variáveis investigadas têm efeitos significativos sobre o nascimento de fetos pré-termo ou de recém-nascidos de baixo peso. Dentre estas situam-se as visitas pré-natais, no que diz respeito à época da primeira e número destas durante o ciclo gestatório.

Wulf e Steck ${ }^{19}$, realizaram interessante estudo na Bavária. Quando o início do tratamento pré-natal se deu após a $21^{\text {a }}$ semana de gestação, o número de recém-nascidos com baixo peso ao nascimento foi significantemente superior ao dos recém-nascidos cujas gestantes tiveram sua primeira consulta entre a $9^{\mathrm{a}}$ e a $12^{\mathrm{a}}$ semana. Da mesma forma a mortalidade perinatal e morbidade neonatal estiveram aumentadas entre as do primeiro grupo. O estudo mostrou ainda que as gestantes com 4 ou menos visitas pré-natais, tiveram mais recém-nascidos de baixo peso e com indices de natimortalidade superiores aos de gestantes que frequentaram o pré-natal 10 ou mais vezes. Como se observa, estes dados tem preocupado os autores no sentido de estabelecerem o momento ideal da primeira consulta pré-natal, assim como o número de visitas ao pré-natalista.

No presente estudo observamos que as gestantes que não realizaram controle antenatal apresentaram uma probabilidade de $36,4 \%$ de chances de terem recém-nascidos com idade gestacional $<37$ semanas. Por outro lado, entre as que fizeram 10 ou mais vistas pré-natais, esta probabilidade foi próxima de zero. Se a gestante realizou 6 ou mais consultas, em apenas $1,5 \%$ dos casos a gestação se interrompeu antes da $32^{\mathrm{a}}$ semana.

As pacientes que não experimentaram qualquer assistência antenatal, ou realizaram no máximo 2 consultas, tiveram uma probabilidade de terem fetos de baixo peso em até $51,2 \%$ das vezes, ao passo que entre aquelas com 10 ou mais visitas pré-natais, esta probabilidade caiu significantemente para 5,8\%. Vale ainda considerar que, se a gestante realizou 6 ou mais consultas, em apenas $1,9 \%$ dos casos o peso fetal foi inferior a $1.500 \mathrm{~g}$.

Mustard e Roos ${ }^{14}$ estudando 13.349 mulheres canadenses que tiveram recém-nascidos vivos, observaram que aquelas que tiveram em média 1,5 visitas pré-natais, apresentaram um maior número de recém-nascidos de baixo peso, e pertenciam a um grupo socio-econômico mais baixo. Embora esta diferença tenha sido significante, porém pequena, atribuem a outros fatores o nascimento destes fetos de baixo peso, tais como tabagismo, pacientes solteiras, e finalmente assistência pré-natal inadequada. Não responsabilizam somente a inadequada assistência pré-natal pelo baixo peso ao nascimento.

Virgi e Cottington ${ }^{18}$ em um estudo realizado nos Estados Unidos, observaram que entre 5.823 gestantes avaliadas, as de raça negra tiveram duas vezes mais parto pré-termo do que as de raça branca, e que aquelas tiveram a idade da gravidez, em média, 1 semana mais curta do que as mulheres brancas, e esta diferença foi significante. Em ambos os grupos, a idade materna (acima de 35 anos e abaixo de 20), o tabagismo, o consumo de álcool, e menos de 9 visitas pré-natais, associaram-se com o aumento das taxas de parto pré-termo. Estes autores referem ter sido o número de visitas pré-natais a variável que mais freqüentemente esteve associada com nascimentos pré-termo.

Aumann e Baird ${ }^{1}$ preconizam uma freqüência mensal de visitas pré-natal, começando quando dos primeiros sinais de gravidez até a $30^{\mathrm{a}}$ semana. Após, uma visita quinzenal até a $36^{\mathrm{a}}$ semana, e semanalmente a partir da $37^{\mathrm{a}}$ semana até o parto. Referem ser benéfico à gestante visitas 
semanais no início da gestação, até a observação dos batimentos fetais, na tentativa de estabelecer a idade gestacional com maior acurácia.

Os resultados do presente estudo demonstram que a precocidade na primeira visita pré-natal associou-se significantemente com o número de gestações de termo. Quando a primeira consulta ocorreu antes da $12^{\mathrm{a}}$ semana, a probabilidade do parto ocorrer no termo foi de $94,9 \%$. Por outro lado, quando a primeira consulta ocorreu após a $28^{\mathrm{a}}$ semana de gestação, em apenas $58,7 \%$ das vezes o parto foi a termo. Merece consideração o fato de a gestante iniciar seu prénatal até a $16^{\mathrm{a}}$ semana e mostrar uma probabilidade de nascimentos antes da $37^{\mathrm{a}}$ semana, de apenas 10,2\%.

O nascimento de fetos de baixo peso associou-se significantemente com o momento da primeira visita antenatal. Se esta ocorreu antes da $12^{\mathrm{a}}$ semana gestacional, a probabilidade do nascimento de fetos de baixo peso foi de $5,1 \%$, ao passo que esta probabilidade aumentou para $43,5 \%$ se a primeira consulta ocorreu após a $28^{\mathrm{a}}$ semana de gravidez. Quando a assistência antenatal foi iniciada até a $16^{\text {a }}$ semana de gravidez, a probabilidade de nascimento de fetos com $<2.500 \mathrm{~g}$ foi de $18,2 \%$, inferior quase duas vezes e meia, se iniciada a assistência após a $28^{\mathrm{a}}$ semana.

Nos Estados Unidos e países da Europa têm sido desenvolvidos programas para prevenir a prematuridade e periodicamente têm merecido a avaliação por parte dos investigadores. Fangman et $\mathrm{al}^{5}$, nos Estados Unidos, observaram que de um grupo de 1.143 gestantes avaliadas, apenas $11,8 \%$ eram de alto risco, sendo que destas $50,2 \%$ tiveram seus filhos prematuros. A associação com nascimentos pré-termo se deu com gestantes jovens, negras, solteiras, e com escolaridade abaixo de 12 anos. Papiernik et $\mathrm{al}^{15}$ na França, estudaram uma população acima 11.000 mulheres num período de 5 anos. Entre as gestantes que precocemente entraram no programa de prevenção do parto pré-termo, a taxa de prematuridade foi de $3,7 \%$, comparada com $8,2 \%$ entre as gestantes que iniciavam seu pré-natal mais tardiamente. Em San Francisco, na California, um programa instituído para prevenir o parto pré-termo reduziu significantemente as suas taxas ${ }^{11}$. Por outro lado, na Australia um programa de suporte social, utilizando visitas domiciliares de enfermeiras treinadas mostrou que no grupo de gestantes estudadas a taxa de partos pré-termo foi de $12,8 \%$ ao passo que no grupo controle foi de $14,9 \%^{2}$. Esta diferença não foi significante, o que levou os autores a não atribuirem relevância a este tipo de suporte dito social.

Não se investigou no presente estudo, outros fatores que pudessem estar relacionados com o nascimento de fetos de baixo peso ou de pré-termo. No entanto julgamos oportuno salientar, que, juntamente com a adequada assistência pré-natal (aqui entendida como início precoce das visitas antenatais, freqüência desejável das mesmas, e atendimento de boa qualidade), uma política de assistência às gestantes de alto risco é fundamentalmente importante para a minimização das taxas de prematuridade. Isto envolve condições de atendimento em níveis diferenciados e regionalizados; educação da população assistida para os riscos que certos fatores exercem sobre o nascimentos pré-termo e o esforço conjunto dos profissionais envolvidos no campo perinatal. Reguero e Crane $^{16}$ estudaram a mortalidade perinatal em New Haven, a sétima cidade mais pobre dos Estados Unidos em renda per capita. Observaram que $21 \%$ da população estudada não tinha qualquer assistência pré-natal ou iniciava o mesmo depois do primeiro trimestre gestacional, e que $71 \%$ dos óbitos perinatais se devia a fetos de baixo peso ao nascimento. Enfatizam que uma assistência pré-natal adequada é o melhor meio de melhorar estas estatísticas.

Hobel et $\mathrm{al}^{8}$ desenvolveram estudo prospectivo randomizado em Los Angeles (West Los Angeles Preterm Birth Prevention Project), onde a educação, o aumento na freqüência de visitas prénatal e intervenções selecionadas profiláticas foram usadas na tentativa de reduzir o nascimento de fetos pré-termo. O estudo compreendeu 1.774 gestantes de alto risco em 8 clínicas de Los Angeles. O grupo de estudo teve uma taxa de $7,4 \%$ de nascimentos de pré-termo, comparada a $9,1 \%$ de um grupo controle. Esta diferença correspondeu a uma redução de 19\% e foi significante. Os dados sugerem que a educação e a maior freqüência de visitas pré-natais, além de intervenções seletivas, podem reduzir o número de fetos prematuros.

Se de um lado uma boa assistência antenatal pode levar a menores índices de partos pré-termo e de recém-nascidos de baixo peso, de outro convém lembrar que as instituições que suportam estes neonatos devem estar preparadas para tal. O número de nascimentos pré-termo pode estar aumentando em função também do avanço científico e tecnológico, necessário e salutar. A manutenção de fetos intra-útero em condições adversas à sua vitalidade, significa aumentar o obituário fetal. Sua retirada prematura e com vitalidade pressupõe chances de sobrevida extrauterina em local apropriadamente equipado. O esforço de uma equipe perinatal deve se concentrar em conhecer a medida e o equilíbrio desejável entre os componentes do binômio probabilidade de vida intra-uterina versus probabilidade de vida extra- 
uterina. Isto definido, as responsabilidades serão divididas, e uma melhor compreensão das necessidades de cada serviço poderá levar ao seu aprimoramento no manejo perinatal das várias situações clínicas. Com isto a morbidade e neomortalidade entre os recém-nascidos pré-termo poderá ser efetivamente minimizada. Fawer et al ${ }^{6}$ referem que o prognóstico de recém-nascidos com peso inferior a $1.000 \mathrm{~g}$ é favorável mas depende da colaboração de obstetras, neonatólogos e da qualidade da assistência perinatal.

Os resultados permitem concluir que a freqüência às consultas pré-natal bem como o momento da primeira visita, se associam significantemente com a idade gestacional e o peso fetal ao nascimento, e podem desempenhar papel importante no nascimento de recém-nascidos pré-termo e de baixo peso.

\section{SUMMARY}

It is universally accepted that prenatal care has a beneficial impact on perinatal outcome. However, it is unclear whether access to early and frequent prenatal care influences the impact of pregnancy complications on birth weight. The objective of the present study was to determine the effectiveness of prenatal care, concerning antenatal visits (number and time of the first one), on gestational age and fetal weight at birth. We assessed prospectively the effect of the antenatal care in a group of 648 infants born consecutively at the University Hospital of Santa Maria, weighing from $<1000$ to $>4000 \mathrm{~g}$, and from $<28$ to $>40$ weeks of gestational age. Preterm delivery ( $<37$ weeks) accounted for $17.7 \%$ of all deliveries, low birth-weight infants $(<2500 \mathrm{~g})$ for $20.5 \%$, and very low birth-weight infants $(<1000 \mathrm{~g})$ for $2.8 \%$. When the first antenatal visit was performed before the 12th week, only $5.1 \%$ of the babies were born with $<37$ weeks of gestational age or weight at birth of $<2500 \mathrm{~g}$. However, when the first visit was after the 28th week, the percentage of preterm delivery was $41.3 \%$ and of birth weight $<2500 \mathrm{~g}$ was $43.5 \%$. A significant association between higher frequency of antenatal visits, early care and decrease in preterm delivery frequency and low birth-weight infants was noted ( $p<0.001)$. We conclude that increase in the number of antenatal visits and early care can reduce the preterm delivery and low-birth weight infant rates.

KEY WORDS: Antenatal care. Prematurity, low bith- weight infants. Fetal weight.

\section{Referências bibliográficas}

1. Aumann GME, Baird MM. Screening for the highrisk pregnancy. In: Knuppel R.A., Drukker J.E. High-risk pregnancy. A team approach. Philadelphia: W. B. Saunders Company, Philadelphia, 1986; p.3-23.

2. Bryce RL, Stanley FJ, Garner JB. Randomized controlled trial of antenatal social support to prevent preterm birth. Br J Obstet Gynaecol 1991; 98: $1001-8$.

3. Chadwick J. Perinatal mortality and antenatal care. Mod Midwife 1994; 4: 18-20.

4. Cohen BB, Friedman DJ, Mahan CM, Lederman R, Munoz D. Ethnicity, maternal risk, and birth weight among hispanics in Massachusets, 198789. Public Health Rep 1993; 108: 363-71.

5. Fangman JJ, Mark PM, Pratt L, Conway KK., Healey ML, Oswald JW, Uden DL. Prematurity prevention programs: an analysis of successes and failures. Am J Obstet Gynecol 1994; 170: 744-50.

6. Fawer CL, Dayer V, Forcada M, Calame A. Prématures extrèmes: quel devenir? Experience lausannoise 1982-1992. Rev Med Suisse Rom 1994; 114: 845-50.

7. Harms K, Osmers R, Kron M, Schill M, Kuhn W, Speer CP, Schroter W. Mortalität unreifer Neugeborener von 1980-1990: Analyse aus dem Perinatalzentrum Göttingen. Z Geburtshilfe Perinatol 1994; 198: 126-33.

8. Hobel CJ, Ross MG, Bermis RL, Coyle W. The West Los Angeles preterm birth prevention project: I. Program impact on high-risk women. Am $\mathbf{J}$ Obstet Gynecol 1994; 170: 54-62.

9. Kunzel W. The birth survey in Germany-education and quality control in perinatology. Eur J Obstet Gynecol Reprod Biol 1994; 54: 13-20.

10. Lopez TR, Ramirez MS, Martinez JDA. Influencia del control prenatal sobre la morbimortalidad materna y perinatal en un centro hospitalario de 2 nivel de atención. Ginecol Obstet Mex 1994; 62: 185-8.

11. Main DM, Richardson DK, Hadley CB, Gabbe SG. Controlled trial of a preterm labor detection program: efficacy and costs. Obstet Gynecol 1989; 74: 873-77.

12. Morais EN, Resener EV, Mottecy CS, Alves AC. Fatores determinantes das taxas de mortalidade perinatal. J Bras Ginecol 1984; 94: 81-5.

13. Morais EN, Wagner A, Deboni L, Daudt CE. Considerações sobre cardiotocografia anteparto e índices de mortalidade perinatal. J Bras Ginecol 1991; 101: 213-17.

14. Mustard CA, Roos N.P. The relathionship of prenatal care and pregnancy complications to 
birthweight in Winnipeg, Canada. Am J Public Health 1994; 84: 1450-1457.

15. Papiernik E, Maine D, Rush D, Richard A. Prenatal care and prevention of preterm delivery. Int $\mathbf{J}$ Gynaecol Obstet 1985; 23: 427-33.

16. Reguero W, Crane M. Project Mother Care: one hospital's response to the high perinatal death rate in New Haven, CT. Public Health Rep 1994; 109: 647-52.

17. Synnes AR, Ling EW, Whitfield MF, Mackinnon M, Lopes L, Wong G, Effer SB. Perinatal outcomes of a large cohort of extremely low gestational age infants (twenty-three to twenty-eight completed weeks of gestation). J Pediatr 1994; 125: 952-60.

18. Virgi SK, Cottington E. Risk factors associated with preterm deliveries among racial groups in a national sample of married mothers. Am J Perinatol 1991; 8: 347-53.

19. Wulf KH, Steck T. The impact of timing and frequency of prenatal visits on the outcome of pregnancy in the perinatal registry of Bavaria 19871988. Eur. J. Obstet. Gynecol Reprod Biol 1994; 57: 79-84. 